\title{
An Inexpensive, Microcomputer-Based, Video Densitometer for Quantitating Thin-Layer Chromatographic Spots'
}

\author{
Thomas S. Ford-Holevinski, Bernard W. AgRanOFF, AND Norman S. Radin \\ Department of Biological Chemistry and Mental Health Research Institute (Department of Psychiatry), \\ University of Michigan, Ann Arbor, Michigan 48109
}

Received December 27, 1982

\begin{abstract}
An inexpensive video densitometer based on an Apple II microcomputer using commercially available components was developed. The device was used to quantitate charred lipid and colored spots on thin-layer chromatography plates. The method is useful for lipid samples weighing 0.1 to $4 \mu \mathrm{g}$ on high-performance plates and 1 to $20 \mu \mathrm{g}$ on ordinary plates. The software automatically corrects for baseline drift due to dirt or nonuniformity in the plate.

KEY WORDS: thin-layer chromatography; quantitation; charring method; microcomputer video densitometer.
\end{abstract}

Several commercial TLC ${ }^{2}$ scanners are curently available. These scanners are typically restricted with respect to the size of TLC spots that can be evaluated, the ability to resolve close spots (due to slit width or noise limitations), and the problem of handling spots that are misaligned. Most of them do not correct for background irregularities, and some require a blank lane for each sample lane, wasting half of every plate. Some of these problems were eliminated by the use of a video camera, coupled to a large computer $(1,2)$, which acted as a densitometer for each intact (nonoverlapping) spot. This approach was developed over the past 8 years by a group in Hungary, which claimed a standard deviation for each analysis of $5-10 \%$. The authors apparently did not determine how much of the variability was instrumental and how much was due to TLC technique.

Recent developments in microcomputers and associated technology indicated to us the possibility that an efficient and economical

\footnotetext{
1 This work was supported by grants from the National Institutes of Health (NS 03192 and NS 13743).

${ }^{2}$ Abbreviations used: TLC, thin-layer chromatography; HPTLC, high-performance TLC.
}

system that would be more versatile than presently available scanners could be devised. The system described here was designed on the basis of the following goals: it should utilize an inexpensive, widely used microcomputer; only commercially available hardware components should be used; a light box and transmittance measurements should be used to simplify the optical system; and the system should be easy to assemble for laboratories lacking shop facilities and computer expertise.

\section{MATERIALS AND METHODS}

Thin-layer chromatography. The samples were applied to E. Merck silica gel 60 plates (without fluorescent indicator) using a modified Camag sample applicator (3) set to make a 1-cm streak. The sample concentrations were chosen so that $40 \mu \mathrm{l}$ would be used for each streak. Brinkmann HPTLC silica gel plates were spotted with $8 \mu$ l of sample as circular spots, using a motorized syringe. All plates were prewashed in the solvent system used for the samples (chloroform:methanol, 96:4). After the plates were run, they were dried, sprayed with charring reagent, and heated at 


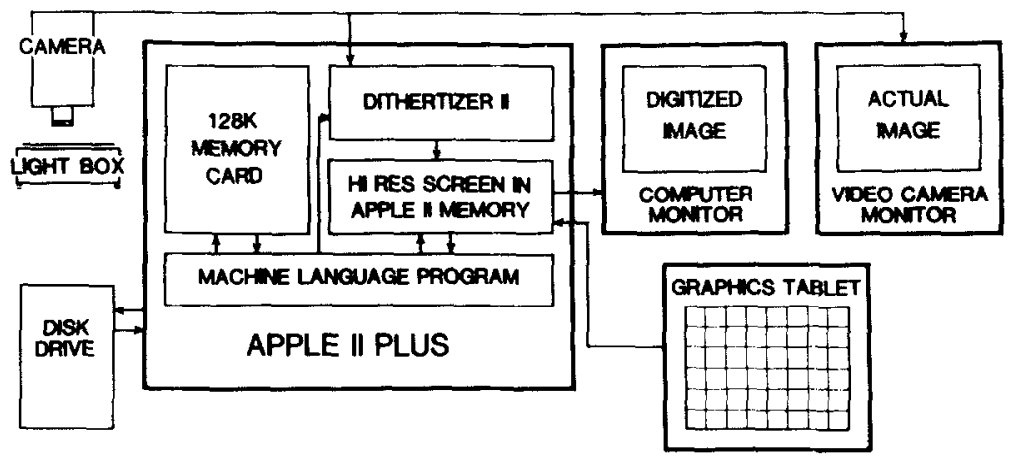

FIG. 1. Schematic drawing of the video densitometer.

$180^{\circ} \mathrm{C}$ on a $1 / 4$-in-thick aluminum plate $(3,4)$. After the plate cooled it was taped to an overlying clean glass plate and stored in a desiccator over $\mathrm{CaCl}_{2}$.

Apparatus. The schematic (Fig. 1) indicates the flow of data through the various components of the system. The TLC plates were placed on a light box containing a circular fluorescent bulb (GLOW-BOX, Model GB 11 8, Instruments for Research and Industry, Cheltenham, Pa.) and covered with black "construction paper" containing a slit that was a little larger than the largest spot. This slit was important because it provided the camera's reference for total blackness.

An RCA black-and-white video camera (Model TC1005/01) fitted with a 1-in Vidicon tube, a 75-mm Wollensak lens set to $f /$ 8 , and $a+2$ close-up lens was placed on a stand over the light box. ${ }^{3}$ The camera was modified to inhibit its automatic light-sensitivity circuits by moving a jumper inside the camera from the automatic to the manual target mode. Light input was interrupted between scans to prevent burning the image into the video tube. The camera and light box were turned on at least $30 \mathrm{~min}$ prior to use. The entire assembly was enclosed in a black wooden box with a black curtain in front.

The video signal was relayed to a standard video monitor for direct visualization. The

\footnotetext{
${ }^{3}$ The greater availability of lenses for the $2 / 3$-in. video tube leads us to suggest the use of that version of this camera.
}

camera was also attached to a Dithertizer II digitizing circuit (Computer Stations Inc., St. Louis, Mo.) which was installed in the Apple II microcomputer. This device uses the process of direct memory access to store the video image in the high-resolution screen portion of the Apple II memory in 1/60 of a second. We also installed a $128 \mathrm{~K}$ memory expansion card (Model 128KDE, Legend Industries Ltd., Pontiac, Mich.), as well as a graphics tablet and 5 1/4-in disk drive (Apple Computer Co., Cupertino, Calif.).

Software. The image produced by the Dithertizer II is binary. This means each picture element ("pixel") in the 280 column by 192 row array is either white or black depending on whether it is above or below a preset threshold limit. The threshold limits are values from 0 to 255 and are set in the Dithertizer II under software control. The video signal is stored as an array of 8-bit values by digitizing the signal at each of the 256 available thresholds and adding the resulting images to the memory expansion card (5). Each image is made up of 53,760 bytes, so the size of our memory expansion allows us to store two complete 8-bit "images" at once. Due to the limitations of the Apple II it is not possible to visualize the various densities as such. This can be overcome, however, by a pseudo-gray scale dithertizing technique (Fig. 2), using algorithms provided with the Dithertizer II card.

After the image is captured, the upper and lower intensity thresholds are set to display the spots on the Apple II high-resolution 


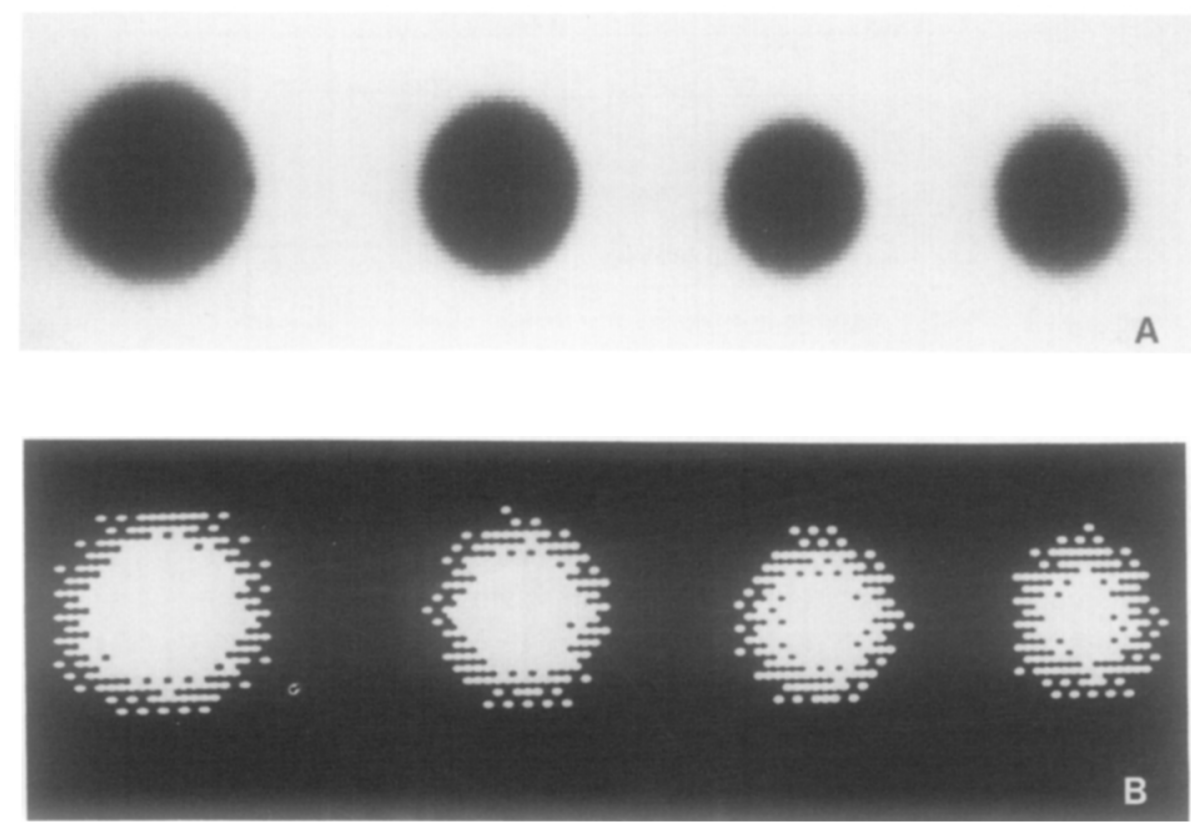

FIG. 2. Video image (A) and dithertized image (B) of spots on an HPTLC plate.

screen. The user then directs a cursor to define a rectangle which completely encompasses the spot. In our case the cursor is generated by the graphics tablet, but this could also be accomplished by a joystick or light pen.

The computer program is designed to find the left and right edges of the spot. A curve depicting the pixel values for each scan line in the rectangle is displayed, the edges of the spot are determined by the computer, and the baseline for the spot is drawn. The edge of the spot is determined by a subtraction algorithm, rather than by the more usual, timeconsuming moving-average algorithm. The threshold value which defines the edge of the spot is set by the user according to the degree of uniformity of the TLC powder. Baseline values are then subtracted from the pixel values within the edges of the spot to correct for background nonuniformity, which can appear in the direction of development. The digital value for the spot is the sum of all the corrected pixel values within the rectangle.

In the interest of speed, the software which performs the image capture, storage, manip- ulation, and display was written in 6502 assembly language.

\section{RESULTS AND DISCUSSION}

Accuracy and reproducibility. Readings were found to drift upward if the system was not turned on at least $30 \mathrm{~min}$ prior to scanning, after which time there was no perceptible drift over a period of several hours. In altering the gain and light-sensitivity controls we removed the video tube's protection from excessive lighting. To avoid burning images into the Vidicon tube, we covered the light source after each image was scanned. We also reduced the lens aperture to $f / 8$, even though this slightly reduced the dynamic range of the spots. Another solution to this problem might be the use of the more costly Ultracon tube version of this camera which is less susceptible to image memory effects.

When a single dark spot ( $15 \mu \mathrm{g}$ of cholesterol developed on a standard TLC plate) was scanned 10 times, we found that the standard deviation was only $0.55 \%$ of the mean. This 
value increased to $1.2 \%$ when the spot was very light $(2.5 \mu \mathrm{g})$. On HPTLC plates, which had smaller spots, a 4- $\mu$ g spot had a standard deviation of $1.2 \%$ of the mean, while a much lighter $(0.38-\mu \mathrm{g})$ spot had a standard deviation equal to $2.3 \%$ of the mean. Scanning 10 spots, each containing $5 \mu \mathrm{g}$ of cholesterol on a single standard TLC plate, tested the variability in the spotting technique, the TLC plate, the charring technique, and the optical system. In this case the standard deviation was $3.2 \%$ of the mean. These values were constant regardless of the magnification used.

Resolution and speed. Slit widths in ordinary mechano-optical scanners are limited in size by the problems of edge diffraction and electrical noise due to the low amount of transmitted light. One advantage of a video scanner is that the pixel size is far from rigid and with proper lenses can be either increased or decreased. In its present configuration, each pixel in our image is approximately $0.17 \mathrm{~mm}$ square. This degree of resolution compares favorably with a currently available laser-beam densitometer (LKB, Stockholm). At this magnification it was possible to scan 40 spots each with a diameter of $5 \mathrm{~mm}$ on an HPTLC plate in approximately $90 \mathrm{~min}$.

Spots which overlap can be resolved to some extent by setting the spot boundary with the cursor control at a point estimated to divide the two spots "equitably." This corresponds to the process used in scanning integration of "dropping a perpendicular from the valley to the baseline." Neither method is as satisfactory as using a solvent which gives good separation.

Range. The curves in Fig. 3 are derived from spots on an HPTLC plate and are plotted as the sum of the digital values for all the pixels within each spot versus the actual weight of the lipid in the spot. The curves for the two compounds follow second-order equations with correlation coefficients of 0.993 for cholesterol and 0.996 for stearoyl sphingosine. Similar curves were produced when 0.94 to $20 \mu \mathrm{g}$ of lipid was run on standard TLC plates. When the HPTLC data were plotted on a log-

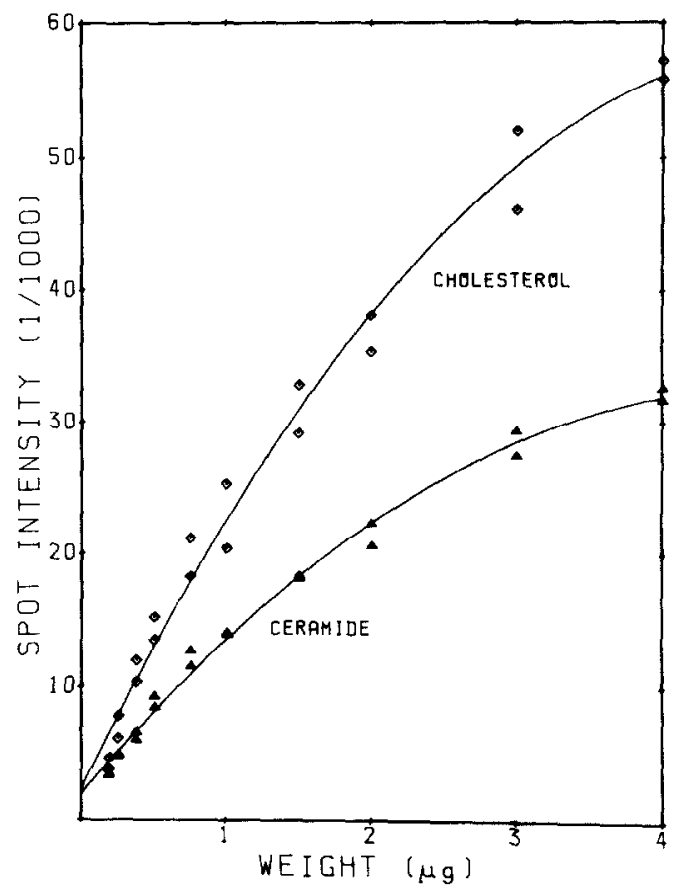

FIG. 3. Analysis of TLC spots produced by charring cholesterol and stearoyl sphingosine on a single HPTLC plate developed with chloroform:methanol, $96: 4$.

log scale the relationship was linear for 0.375 to $4 \mu \mathrm{g}$ of cholesterol $(R=0.991)$ and for 0.188 to $4 \mu \mathrm{g}$ of stearoyl sphingosine ( $R$ $=0.993$ ).

The differences in the spot intensities of the two compounds may be due to the greater area of the (faster-moving) cholesterol spots. In this system, as in other systems studied (6), marked differences in intensity are obtained with starting spots of different dimensions. For example, $5 \mu \mathrm{g}$ of oil red $\mathrm{O}$ yielded intensities of 166,130 (round spot, $0.5-\mathrm{cm}$ diameter), 229,660 (1-cm streak), and $271,020(1.5-\mathrm{cm}$ streak).

When the data are plotted as a percentage of the maximal value, the curves for the two compounds are almost exactly the same. This suggests that the internal standard technique would be practical for our video system. In this method, a standard curve is obtained by including one of at least three different levels of a nonoverlapping substance in all of the 
unknown samples. An additional set of duplicate lanes would be used for standard mixtures composed of the substances to be determined in the unknowns, applied at a level near the upper end of the linear region. Thus several different compounds, if they occur in the unknowns at concentrations within the linear region, can be determined on the same plate with only two lanes of standards.

Filters and light sources. We found that this device would also work with oil red $\mathrm{O}$, a colored compound, as well as the charred spots. The common practice of using monochromatic light for TLC scanning may thus not always prove necessary. It is likely that the densitometer would work with other colored compounds and, with an ultraviolet light source and filter for the lens, for fluorescent compounds as well.

It should be pointed out that the problems of nonuniformity in light intensity of the source and sensitivity of different regions of the video camera were greatly reduced in our procedure by examining each spot at the same point in the field of vision. The time required for plate analysis could be greatly reduced by scanning and quantitating many spots at one time. The reliability of this technique, however, is likely limited by the quality of the video tube used. Subtracting the image of a blank plate was not of great value for our system.

Storage of charred plates. When plates were covered with a clean glass plate shortly after charring and kept in a desiccator over $\mathrm{CaCl}_{2}$ when not being scanned, the readings did not change over a period of several days.

Cost. Although the system described here does not have the resolution or speed of commercially available minicomputer-based image processors, it costs only a fraction as much. The total cost of the system is less than $\$ 4000$ if a joystick is substituted for the graphics tablet to indicate the rectangle to be scanned and if a $64 \mathrm{~K}$ memory board is used. The $128 \mathrm{~K}$ board, however, might be preferable if one needed to perform additional image processing (e.g., subtraction of background images to correct lighting nonuniformity). The second monitor was useful in developing the program and in centering the spot in the lighted area, but one could operate the system with a single monitor. It should be noted that the inexpensive mechano-optical scanners available do not include electronic integrators for the actual quantitation step.

Other uses. The device we have described could also be used to analyze radioautographic spots and enzyme-linked immunoassay reactions or to count grains on exposed film. A somewhat similar system which used the Apple II for the quantitation of two-dimensional electrophoretograms was recently developed by Mariash et al. (7). Their system, however, is limited to 8192 pixels, each with 64 gray levels, and apparently does not display the digitized image.

\section{ACKNOWLEDGMENT}

We thank Inez Mason for her competent technical assistance.

\section{REFERENCES}

1. Dévényi, T. (1976) Acta Biochim. Biophys. Acad. Sci. Hung. 11, 1-10.

2. Dévényi, T., and Pongor, S. (1980) Hung. Sci. Instrum. 50, 3-8.

3. Radin, N. S., Deshmukh, G. D., Selvam, R., and Hospattankar, A. V. (1982) Biochim. Biophys. Acta 713, 474-478.

4. Fewster, M. E., Burns, B. J., and Mead, J. F. (1969) J. Chromatogr. 43, 120-126.

5. Ford-Holevinski, T. S., Radin, N. S., and Agranoff, B. W. (1983) Submitted for publication.

6. Sherma, J., Touchstone, J. C., and Dobbins, M. F. (1979) in Densitometry in Thin Layer Chromatography (Touchstone, J. C., and Sherma, J., eds.), pp. 95-120, Wiley, New York.

7. Mariash, C. N., Seelig, S., and Oppenheimer, J. H. (1981) Anal. Biochem. 121, 388-394. 